\title{
Dorsal root ganglia NR2B-mediated Epac1-Piezo2 signaling pathway contributes to mechanical allodynia of bone cancer pain
}

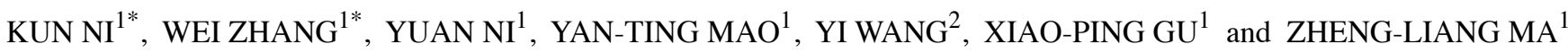 \\ Departments of ${ }^{1}$ Anesthesiology and ${ }^{2}$ Neurosurgery, Affiliated Drum Tower Hospital of \\ Medical School of Nanjing University, Nanjing, Jiangsu 210008, P.R. China
}

Received August 29, 2020; Accepted February 4, 2021

DOI: $10.3892 / \mathrm{ol} .2021 .12599$

\begin{abstract}
Mechanical allodynia is a painful perception of mechanical stimuli and one of the typical symptoms in bone cancer pain (BCP). Previous studies have revealed that mice and humans lacking mechanically activated Piezo2 channels do not sense mechanical stimuli. However, the underlying mechanism of Piezo2 in BCP has not been well established. The aim of the present study was to investigate whether exchange protein directly activated by cAMP 1 (Epac1) mediated Piezo2 signaling pathway may be responsible for the mechanical allodynia of BCP and whether N-methyl-D-aspartic acid (NMDA) receptor subunit $2 \mathrm{~B}$ (NR2B) is involved in the pathway. In the present study, a BCP model was established in $\mathrm{C} 3 \mathrm{H} / \mathrm{HeJ}$ mice by intramedullary injection of osteosarcoma cells. The results of the mechanical allodynia test demonstrated a markedly decreased paw withdrawal mechanical threshold in BCP mice, accompanied by a significant increase in Epac1, NR2B proteins and Piezo2 mRNA expression levels in the ipsilateral dorsal root ganglion (DRG). Compared with the sham group, intrathecal Epac1 antisense oligodeoxynucleotides (Epac1-ASODN) effectively ameliorated the mechanical allodynia and decreased the expression levels of NR2B and Piezo2 in the tumor group. Pretreatment of naïve mice with a NR2B antagonist prevented the aggravation of mechanical allodynia and DRG Piezo2 levels induced by an Epac1 agonist. However, the NR2B agonist-induced increase in Piezo2 expression levels was not reversed by pretreatment with Epac1-ASODN. In conclusion, the results of the present study demonstrated that NR2B, which is a crucial downstream regulator of Epac1, may mediate the Epac1-Piezo2 pathway
\end{abstract}

Correspondence to: Dr Zheng-Liang Ma, Department of Anesthesiology, Affiliated Drum Tower Hospital of Medical School of Nanjing University, 321 Zhongshan North Road, Nanjing, Jiangsu 210008, P.R. China

E-mail: mazhengliang1964@nju.edu.cn

*Contributed equally

Key words: bone cancer pain, mechanical allodynia, dorsal root ganglia, Epac1-Piezo2 pathway, NR2B contributing to the development of the mechanical allodynia of $\mathrm{BCP}$. The present study may enrich the theoretical knowledge of the mechanical allodynia of $\mathrm{BCP}$ and provide a potential analgesic strategy for clinical treatment.

\section{Introduction}

Bone cancer pain (BCP) is one of the most common and intractable symptoms affecting patients with primary or metastatic bone cancer, and is characterized by allodynia or hyperalgesia (1). Despite understanding the effects of $\mathrm{BCP}$ on the health-associated quality of life, BCP remains inadequately controlled in numerous patients (2), due to limited breakthroughs made in understanding the underlying mechanisms and developing therapeutics for BCP (3).

Piezo2, which is a member of the evolutionarily conserved mechanosensitive Piezo channel family, is a rapidly adapting mechanically-activated nonselective cation channel that is most prominently expressed in sensory tissues, especially in the dorsal root ganglion (DRG) neurons (4). Most rapidly adapting mechanically-activated currents in DRG neuronal cultures are absent in Piezo2-conditional knockout mice (5). Mice lacking Piezo2 not only exhibit impaired nociceptive responses to mechanical stimuli in the sensory neurons, but also do not react to capsaicin-induced inflammation or spared nerve injury (6). In addition, individuals with loss-of-function mutations in Piezo2 fail to develop sensitization and reactions to mechanical stimuli following skin inflammation (7). These studies in mice and human patients indicate the essential role of Piezo2 in mechanical allodynia.

Numerous studies have demonstrated that cyclic adenosine monophosphate (cAMP) signaling serves an important role in regulating pain sensitivity through the exchange protein directly activated by cAMP (Epac), which has two isoforms, namely Epac1 and Epac2 (8-10). Intraplantar injection of the Epac agonist 8-pCPT induces long-lasting mechanical allodynia (11); however, nerve damage-induced mechanical allodynia is markedly decreased in Epacl $^{-/}$mice (12). Eijkelkamp et al (12) have reported that DRG Epac1 potentiation of Piezo2-mediated mechanotransduction contributes to mechanical allodynia. Together, these data suggest that the Epac1-Piezo2 signaling pathway may contribute to the establishment of mechanical allodynia in multiple forms of pain (12). However, to the best of our knowledge, there are currently no reports on the role of Piezo2 in BCP. 
The present study used a mouse model of osteosarcoma-associated BCP to determine whether the DRG Epac1-Piezo2 signaling pathway may be responsible for the mechanical allodynia of BCP and to further examine whether $\mathrm{N}$-methyl-D-aspartic acid (NMDA) receptor subunit $2 \mathrm{~B}$ (NR2B), which is as an important regulatory factor, is involved in this mechanism.

\section{Materials and methods}

Animals. Male C3H/HeJ mice, weighing 20-25 g (4-6 weeks old), were provided by the Vital River Laboratory Animal Technology Company. The animals were housed in a temperature-controlled room $\left(21 \pm 1^{\circ} \mathrm{C}\right)$ with a 12 -h dark/light cycle and were provided ad libitum food and water. Mice were used for behavioral experiments ( $\mathrm{n}=8 \mathrm{mice} / \mathrm{group})$, western blotting and reverse transcription-quantitative (RT-q) PCR assays $(n=3$ mice/group). All protocols were approved by the Animal Care and Use Committee of Nanjing University (Nanjing, China) and followed the relevant ethical guidelines for the use of laboratory animals (13).

Cell culture and implantation. Osteosarcoma NCTC 2472 cells (cat. no. 2087787; American Type Culture Collection) were incubated in NCTC 135 medium (Sigma-Aldrich; Merck KGaA) supplemented with $10 \%$ horse serum (Gibco; Thermo Fisher Scientific, Inc.) at $37^{\circ} \mathrm{C}$ in $5 \% \mathrm{CO}_{2}$ and $95 \%$ air.

The mouse model of BCP was established according to previous studies $(14,15)$. Mice were intraperitoneally injected with $50 \mathrm{mg} / \mathrm{kg}$ pentobarbital sodium (1\% in normal saline). Gonarthrotomy was performed to expose the condyles of the distal femur. A 30-gauge needle was used to perforate the cortex, and $1 \times 10^{5}-1 \times 10^{6}$ NCTC 2472 cells in $20 \mu 1 \alpha$-minimal essential medium ( $\alpha$-MEM; Gibco; Thermo Fisher Scientific, Inc.) was injected into the intramedullary space of the femur with a microsyringe. The femurs of the mice in the sham group were injected with $20 \mu \mathrm{l} \alpha$-MEM alone. The injection hole was sealed with bone wax, and the wound was sutured closed.

Drug preparation and injection. The Epac activator 8-pCPT was obtained from the Biology Life Science Institute, and the NMDA receptor agonist NMDA and the NR2B-selective antagonist ifenprodil were purchased from Sigma-Aldrich; Merck KGaA. The Epac1 antisense (AS) or mismatch (MM) oligodeoxynucleotides (ODNs) were obtained from Thermo Fisher Scientific, Inc. The following Epac1-ODNs were used (12): ASODN, 5'-AACTCTCCACCCTCTCCCA-3'; and MMODN, 5'-ACATTCCACCCTCCTCCAC-3'. All reagents were dissolved in normal saline.

Mice received an intraplantar injection of $2.5 \mu \mathrm{l} 8$-pCPT (12.5 nmol) (12) or intrathecal administration of Epac1-ODN $(10 \mu \mathrm{g} / 5 \mu \mathrm{l})(12)$, NMDA (1 nmol/5 $\mu \mathrm{l})(16)$ and ifenprodil (5 $\mu \mathrm{g} / 5 \mu \mathrm{l})(17)$.

Mechanical allodynia tests. Mechanical allodynia was evaluated as previously described $(14,18)$. Mice were individually placed into a transparent plastic chamber with a metal mesh bottom and allowed to habituate for $30 \mathrm{~min}$ prior to testing. Paw withdrawal mechanical threshold (PWMT) of the right hind paw was measured using von Frey filaments
$(0.16,0.40,0.60,1.00,1.40$ and $2.00 \mathrm{~g})$. The filaments were used to vertically push against the plantar surface of the right hind paw. A positive response was brisk withdrawal or licking of the stimulated hind paw. Each mouse was tested five times per stimulus force. The lowest filament that evoked $\geq 3$ positive reactions was set as the PWMT value.

Western blotting. Mice were anesthetized with $50 \mathrm{mg} / \mathrm{kg}$ sodium pentobarbital (1\% in normal saline) and sacrificed by cervical dislocation. The right lumbar DRG L2-L5 segments were rapidly removed and stored in liquid nitrogen. The tissue samples were homogenized with $10 \mu \mathrm{l} / \mathrm{mg}$ RIPA lysis buffer (cat. no. C1053; Applygen Technologies, Inc.) and centrifuged $\left(4^{\circ} \mathrm{C}\right)$ at $25,600 \times \mathrm{g}$ for $10 \mathrm{~min}$. The protein concentration was determined using a BCA Protein Assay kit (Nanjing KeyGen Biotech Co., Ltd.). The protein samples (50 $\mu \mathrm{g} / \mathrm{sample})$ were separated by $10 \%$ SDS-PAGE (Bio-Rad Laboratories, Inc.) and transferred to a PVDF membrane (EMD Millipore). The membrane was blocked with a $5 \%$ non-fat milk solution at room temperature for $1 \mathrm{~h}$ and subsequently incubated with the following primary antibodies at $4^{\circ} \mathrm{C}$ overnight: Rabbit anti-Epac1 (1:1,000; cat. no. ab124162; Abcam), anti-phosphorylated (p-)NR2B (1:1,000; cat. no. ab65783; Abcam) and mouse anti- $\beta$-actin (1:2,000; cat. no. TA-09; OriGene Technologies, Inc.). The next day, the blots were incubated at room temperature with goat anti-rabbit $(1: 2,000$; cat. no.ZB-2301) or goat anti-mouse (1:2,000; cat. no.ZB-2305) (both from OriGene Technologies, Inc.) secondary antibodies conjugated with horseradish peroxidase. Immunoblots were developed using an enhanced chemiluminescence detection system [Hangzhou MultiSciences (Lianke) Biotech Co., Ltd.) and quantified using Quantity One V4.62 software (Bio-Rad Laboratories, Inc.). $\beta$-actin was used as the loading control.

$R T$ - $q P C R$. Mouse right lumbar DRG (L2-L5) segments were isolated and frozen in liquid nitrogen. Total RNA was isolated and purified using TRIzol ${ }^{\circledR}$ reagent (Beijing CWbio). The RNA concentration was measured using an ultraviolet-visible spectrometer (Shanghai Meipuda Instrument Co., Ltd.). Reverse transcription was performed using an RT-PCR kit (Takara Biotechnology Co., Ltd), and qPCR was performed using a SYBR $^{\circledR}$ PrimeScript qRT-PCR kit (Takara Biotechnology Co., Ltd.) according to the manufacturer's protocol. The following primer pairs were used (12): Piezo2 forward, 5'-ATTGGCTGG AGGAGAAA-3' and reverse, 5'-GAAGGTGGAAGAGTG GGAGT-3'; $\beta$-actin forward, 5'-AAGAAGGTGGTGAAG CAGG-3' and reverse, 5'-GAAGGTGGAAGAGTGGGAGT-3' (Sangon Biotech Co., Ltd.). The thermocycling conditions were as follows: $95^{\circ} \mathrm{C}$ for $10 \mathrm{~min}$, followed by 40 cycles of $95^{\circ} \mathrm{C}$ for $10 \mathrm{sec}, 60^{\circ} \mathrm{C}$ for $30 \mathrm{sec}$ and $72^{\circ} \mathrm{C}$ for $30 \mathrm{sec}$. The relative levels of mRNA were calculated using the $2^{-\Delta \Delta \mathrm{Cq}}$ method and optimized with a standard curve to confirm specificity (19). The change in the fluorescence of the amplification product was used to determine the amount of original template.

Statistical analysis. Data are presented as the mean \pm SD. Repeated measures analysis of variance (ANOVA) was performed to determine the overall differences at each time point for PWMT. One-way ANOVA was used to detect differences in the expression levels of proteins or mRNAs among all 
experimental groups. Post hoc analysis was performed using the LSD test for multiple comparisons in order to determine the sources of difference. $\mathrm{P}<0.05$ was considered to indicate a statistically significant difference.

\section{Results}

Bone cancer-induced mechanical allodynia and DRG Epac1, NR2B and Piezo2 expression levels over time. To assess mechanical allodynia in the BCP mouse model, PWMT of the right hind limb was measured on the day before modeling (day-1) as the baseline value, and at days 4, 7, 10 and 14 after modeling. No significant differences were observed in the baseline PWMT (day-1) between the tumor-bearing and sham-operated mice. In the sham group, the PWMT displayed a significant decrease on day 4 and recovered to the pre-modeling level on day 7. Although the tumor-bearing mice also exhibited a marked decrease in PWMT on day 4, it subsequently continued to decrease over time until day 14 (Fig. 1A). These results demonstrated the successful establishment of mechanical allodynia in the BCP mouse model.

To examine the expression levels of Epac1, NR2B and Piezo2 in the DRG of the BCP model mice, western blotting and RT-qPCR assays were performed. Compared with those on day-1, the protein expression levels of Epac1 and p-NR2B in the tumor-bearing mice were increased on day 4 post-modeling and reached the highest levels on day 14 (Fig. 1B). The mRNA expression levels of Piezo2 were significantly increased on day 7 compared with those on day-1 and were gradually upregulated over time until day 14 (Fig. 1C). Thus, the increasing tendency of protein or mRNA expression, which coincided with the PWMT changes, suggested that DRG Epac1, NR2B and Piezo2 may be involved in the pathogenesis and development of mechanical allodynia in BCP.

Effects of intrathecal administration of Epac1-ASODN on bone cancer-induced mechanical allodynia and protein or $m R N A$ expression. Based on the aforementioned results, the present study further assessed the effects of intrathecal injections of Epac1-ODN. Tumor-bearing mice were randomly divided into three groups on day 14 post-modeling and received Epac1-ASODN, Epac1-MMODN or saline once daily between days 14 and 16. PWMT was measured on day 14 prior to administration and days 17,19 and 21 post-modeling.

As demonstrated in Fig. 2A, mice in the vehicle (treated with saline) and Epac1-MMODN groups exhibited a stable PWMT from day 14 throughout the experiment. In contrast, Epac1-ASODN-treated mice exhibited an increase in PWMT on day 17 , and this effect was maintained until day 21 . In addition, Epac1-ASODN significantly downregulated the protein expression levels of DRG Epac1 and p-NR2B, as well as the Piezo 2 mRNA expression levels, in a time-dependent manner (Fig. 2B and C). These results suggested that the DRG Epac1-Piezo2 signaling pathway may be required for the mechanical allodynia of BCP, and NR2B may be involved in the underlying mechanism.

Effects of administration the Epacl or NR2B agonists on mechanical allodynia and protein or mRNA expression. To elucidate the association between NR2B and the Epac1-Piezo2
A
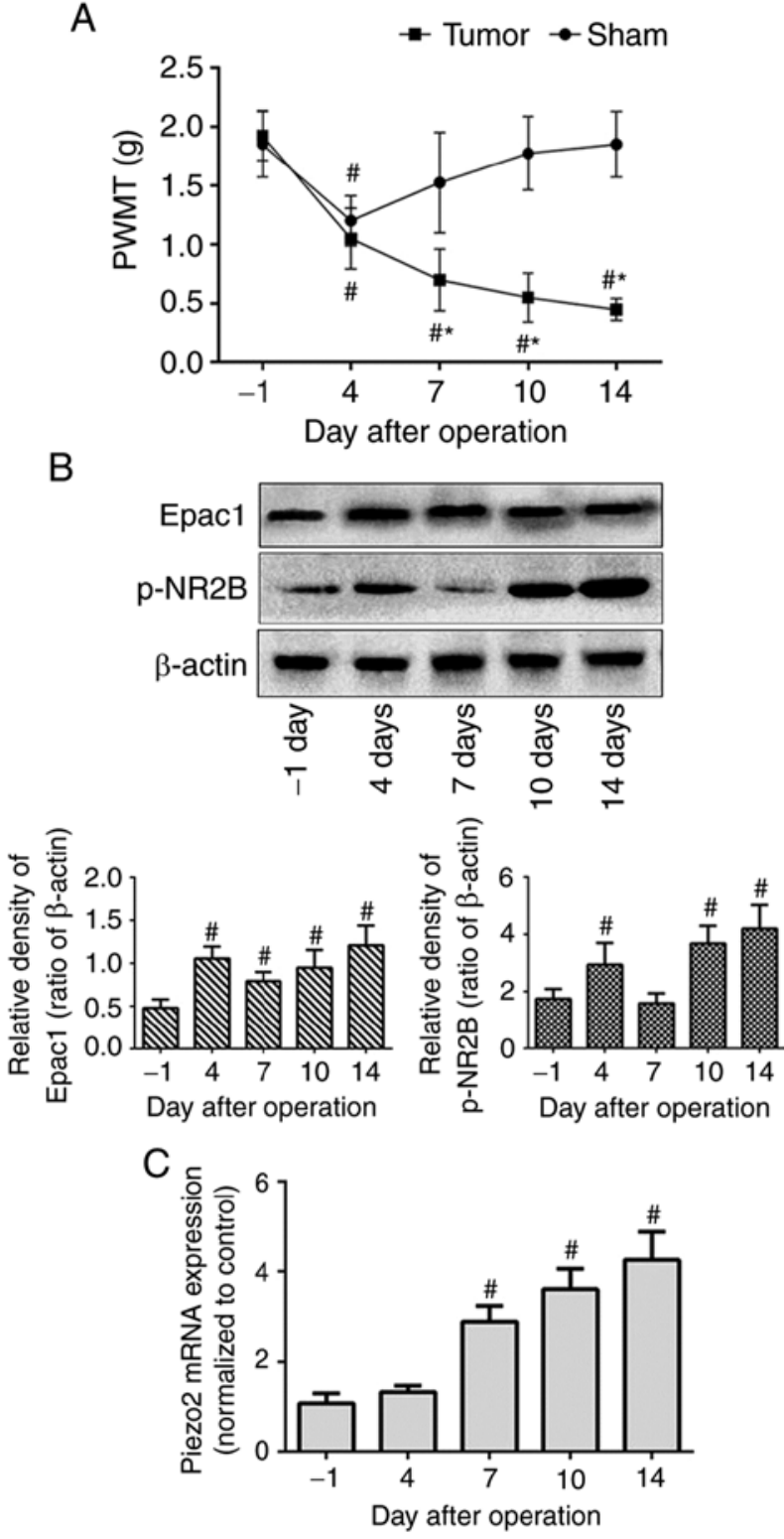

Figure 1. Aggravation of mechanical allodynia and upregulation of DRG Epac1, NR2B and Piezo2 expression in mice with BCP over time. Intra-femur implantation of osteosarcoma cells induced mechanical allodynia in the ipsilateral hind paw. (A) PWMT was measured at the day before modeling (day-1), and days 4, 7, 10 and 14 in sham and tumor group mice (n=8/group). (B and C) Expression level of Epac1, p-NR2B and Piezo2 of the ipsilateral lumbar DRG L2-L5 segments were detected with western blotting ( $\mathrm{n}=3$ /group) or reverse transcription-quantitative $\mathrm{PCR}$ ( $\mathrm{n}=3$ /group) at the corresponding time point. Data represents means $\pm \mathrm{SD}$. ${ }^{\#} \mathrm{P}<0.05$ vs. day- 1 ; ${ }^{*} \mathrm{P}<0.05$ vs. sham group at each time point. BCP, bone cancer pain; DRG, dorsal root ganglion; PWMT, paw withdrawal mechanical threshold.

signaling pathway, the present study first tested whether selective activation of Epac1 or NR2B increased mechanical allodynia. The Epac agonist 8-pCPT was injected into the right hind paw of normal mice as previously described (12). Compared with that in the vehicle group treated with saline, 8-pCPT significantly decreased the PWMT in the ipsilateral hind paw of normal mice $\sim 5$ min after administration, and the lowest PWMT values were observed at $30 \mathrm{~min}$. Similarly, intrathecal injection of NMDA decreased the PWMT in the right hind paw $\sim 5$ min after injection, with the lowest values observed at $15 \mathrm{~min}$ post-injection (Fig. 3A). In addition to 


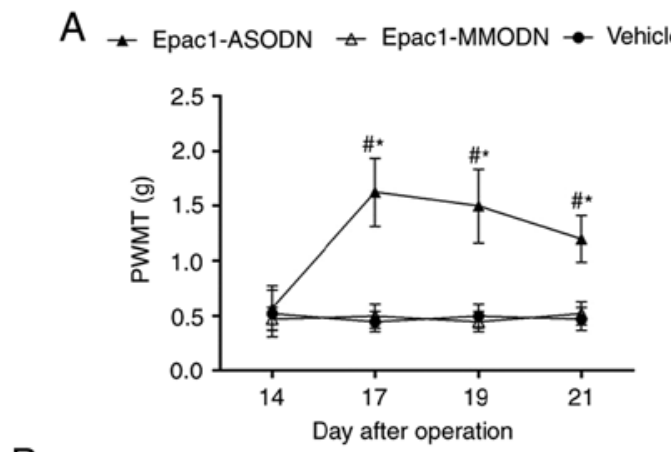

B
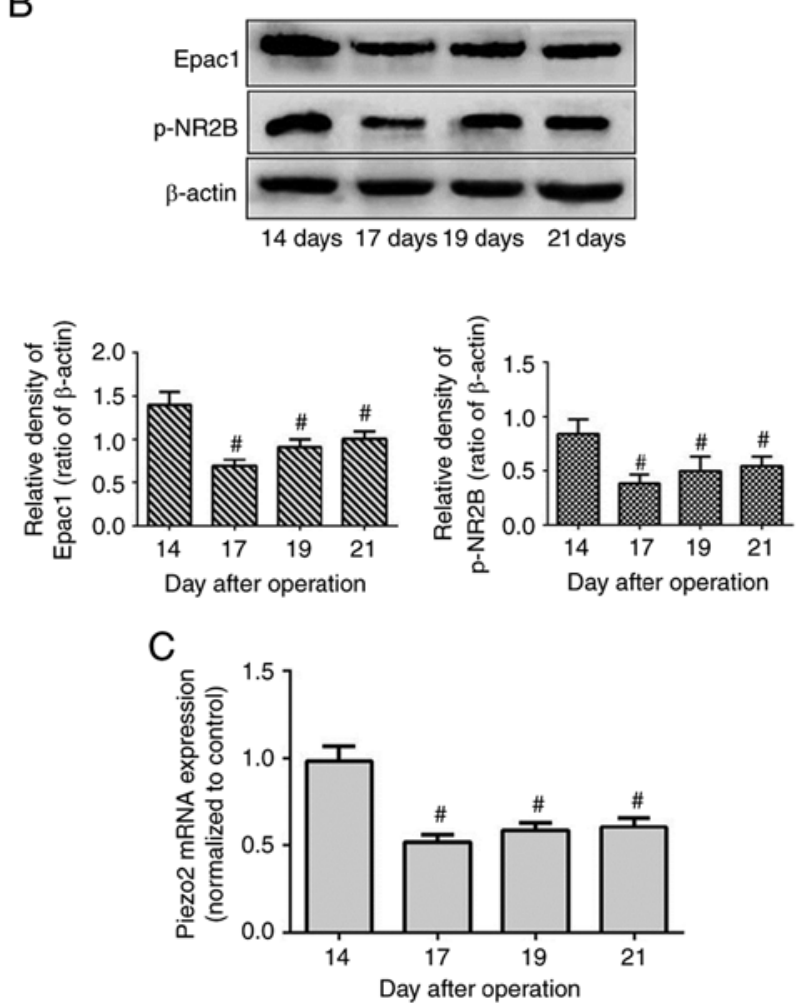

Figure 2. Alleviation of mechanical allodynia and downregulation of DRG Epac1, NR2B and Piezo2 expression by intrathecal injection of Epac1-ASODN in mice with BCP. Three groups of tumor-bearing mice ( $\mathrm{n}=8$ /group) received intrathecal saline $(5 \mu \mathrm{l})$, Epac1-ASODN $(10 \mu \mathrm{g} / 5 \mu \mathrm{l})$ and Epac1-MMODN $(10 \mu \mathrm{g} / 5 \mu \mathrm{l})$ once a day for three consecutive days (from day 14 to 16 after operation). (A) PWMT was measured on day 14 before administration and days 17, 19 and 21 post-operation. (B and C) Expression level of Epac1, p-NR2B and Piezo2 of the ipsilateral lumbar DRG L2-L5 segments were detected with western blotting ( $n=3 /$ group) or reverse transcription-quantitative PCR ( $\mathrm{n}=3$ /group) at the corresponding time point. Data represents means $\pm \mathrm{SD}$. ${ }^{\#} \mathrm{P}<0.05$ vs. day 14 before administration; ${ }^{*} \mathrm{P}<0.05$ vs. vehicle group at each time point. ASODN, antisense oligodeoxynucleotides; MMODN, mismatch oligodeoxynucleotides; BCP, bone cancer pain; DRG, dorsal root ganglion; PWMT, paw withdrawal mechanical threshold.

increasing the expression levels of the tested proteins (Fig. 3B), 8-pCPT and NMDA also increased the ipsilateral DRG Piezo2 mRNA expression levels in normal mice (Fig. 3D). These results demonstrated that not only Epac1, but also NR2B was involved in the regulation of the Piezo2-mediated mechanical allodynia.

Effects of intrathecal pretreatment on the Epacl or NR2B agonist-induced mechanical allodynia and protein or $m R N A$ expression. To further clarify the association between Epac1 and NR2B in the regulation of Piezo2, normal mice were intrathecally pretreated with ifenprodil or Epac1-ASODN prior to the injection of 8-pCPT or NMDA. Compared with the control group (pretreated with saline $5 \mathrm{~min}$ before 8-pCPT), intrathecal pretreatment with ifenprodil prevented the decrease in the PWMT (Fig. 4A) and the increase in ipsilateral DRG Piezo2 mRNA expression levels (Fig. 4B) induced by 8-pCPT, but had no effects on Epac1 expression levels (Fig. 4C). In contrast, intrathecal injection of NMDA induced a decrease in the PWMT (Fig. 4A) and elevated the levels of Piezo2 and p-NR2B expression in normal mice (Fig. 4B and D), which was not affected by pretreatment with Epac1-ASODN. These results suggested that NR2B, which is a critical downstream regulator of Epac1, may be involved in the regulation of mechanical allodynia by the Epac1-Piezo2 signaling pathway.

\section{Discussion}

Mechanical allodynia, which is the painful perception of mechanical stimuli, is one of the typical symptoms in clinical BCP $(20,21)$. The DRG serves an essential integratory role in the modulation of the peripheral and central sensory processing of pain, and is an excellent target for pain research and therapy (22). The results of the present study demonstrated that the mechanical allodynia induced by bone cancer in a mouse model was associated with increased expression levels of DRG Epac1, NR2B and Piezo2. The selected Epac1-ASODN markedly increased the PWMT and decreased the expression levels of Epac1, NR2B and Piezo2 in the mouse model of BCP. Pretreatment with the NR2B antagonist prevented the aggravation in mechanical allodynia and DRG Piezo2 expression levels induced by the Epac1 agonist. However, the NR2B agonist-induced increase in the Piezo 2 expression levels was not reversed by pretreatment with Epac1-ASODN. Overall, the results of the present study may enrich the theoretical knowledge of the mechanical allodynia of BCP and provide a potential analgesic strategy for clinical treatment.

Epac1 is one of the primary downstream sensors of cAMP, which is an intracellular signaling messenger that alters the pain threshold $(23,24)$. In vitro cultures of sensory neurons, the activation of Epac1 enhances the Piezo2 currents, whereas specific activation of other cAMP sensors PKA or Epac2 does not affect Piezo2 sensitivity (12). In addition, mechanical allodynia induced by an intraplantar injection of an Epac agonist is attenuated by Piezo 2 knockdown (12). These results suggest that Piezo2 serves a crucial role in the development of mechanical allodynia, which involves Epac1. The present results demonstrated that an intrathecal injection of Epac1-ASODN effectively alleviated mechanical allodynia and decreased the DRG Piezo2 expression levels in a mouse model of BCP, which may be due to the inhibition of the Epac1-dependent Piezo2 pathway.

Previous studies using cryo-electron microscopy have revealed that $\mathrm{Piezo} 2$ is a large membrane protein with a complicated structure that contains $>30$ potential phosphorylation sites $(25,26)$. The potentiation of Piezo2 currents is associated with the increases in cytosolic $\mathrm{Ca}^{2+}$ after Epac1 activation (12). These studies indicate that phosphorylation may be the mechanism by which Epac1 regulates Piezo2 activity. However, based on the structure and 

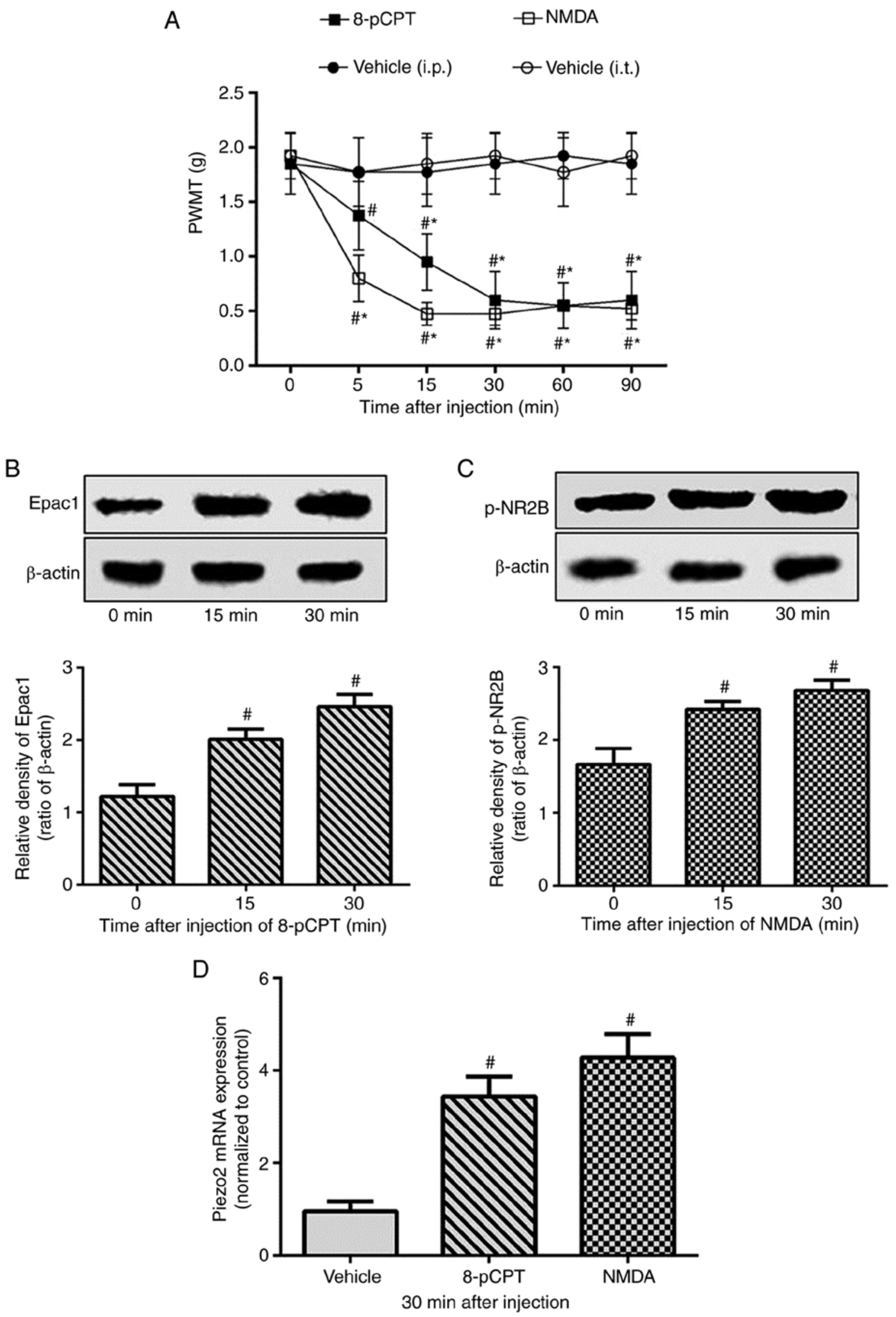

Figure 3. Exacerbation of mechanical allodynia and upregulation of DRG Piezo2 expression by selective activation of Epac1 or NR2B in normal mice. Normal mice were randomly divided into three groups (n=8/group) and received intrathecal (i.t.) saline $(5 \mu 1)$ and NMDA $(1 \mathrm{nmol} / 5 \mu 1)$, or intraplantar saline $(5 \mu 1)$ and 8-pCPT (12.5 nmol/5 $\mu \mathrm{l})$, respectively. (A) PWMT was measured at 0 (before injection), 5, 15, 30, 60 and 90 min after intrathecal or intraplantar injection. (B-D) The expression level of the right lumbar DRG L2-L5 segments of Epaclin 8-pCPT group, p-NR2B in NMDA group and Piezo2 mRNA in both groups were detected at the time of 0,15 or 30 min after injection. Data represents means $\pm \mathrm{SD}$. ${ }^{\#} \mathrm{P}<0.05$ vs. 0 min; ${ }^{*} \mathrm{P}<0.05$ vs. vehicle group at each time point. DRG, dorsal root ganglion; PWMT, paw withdrawal mechanical threshold. 
A

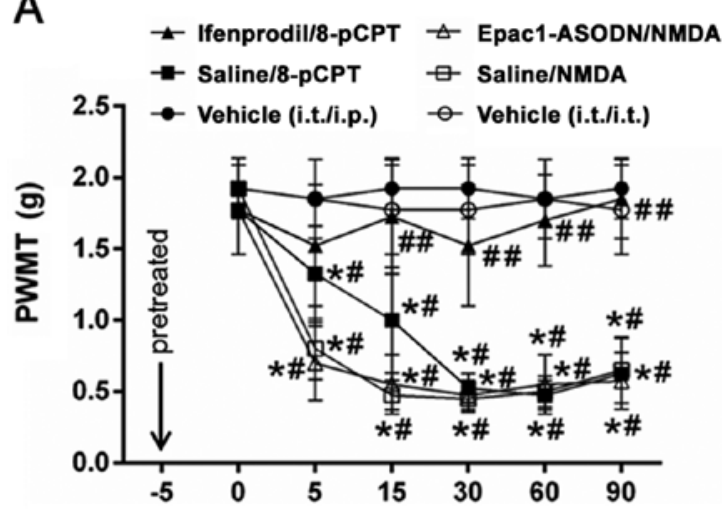

C
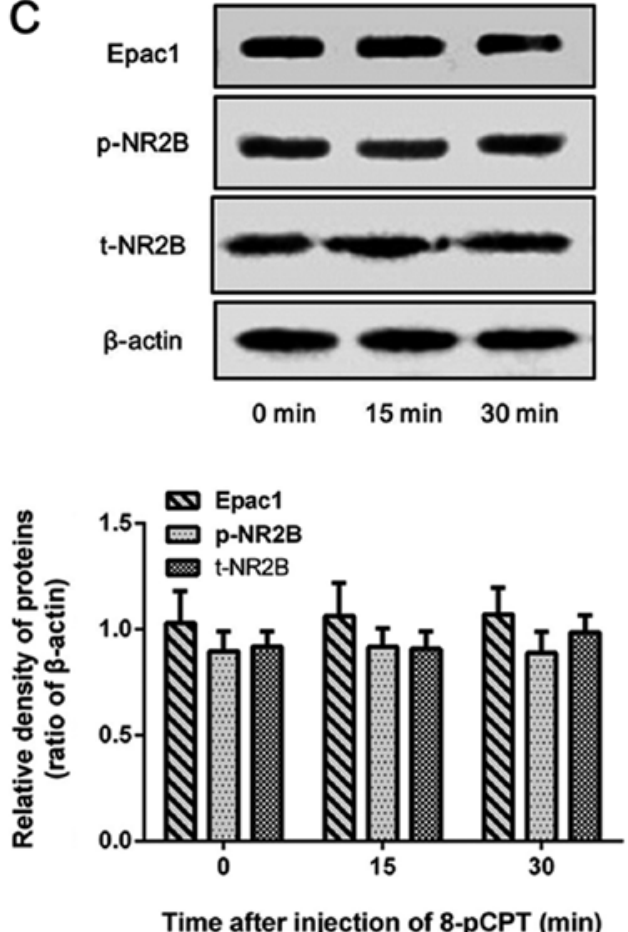

B

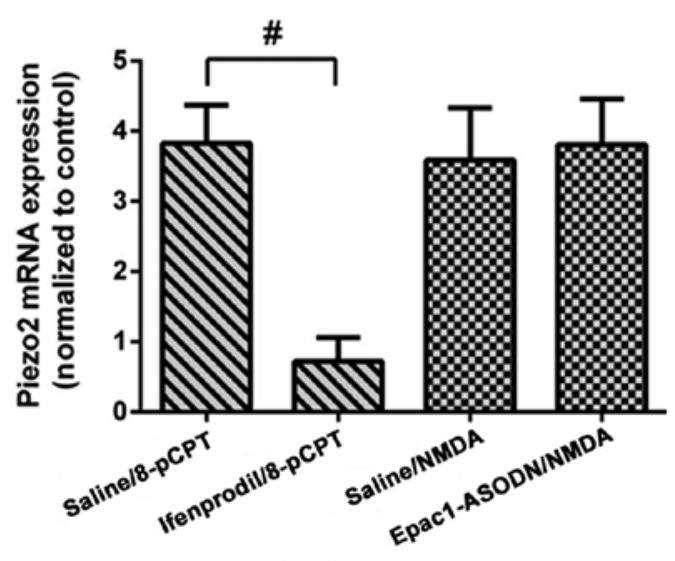

$30 \mathrm{~min}$ after injection
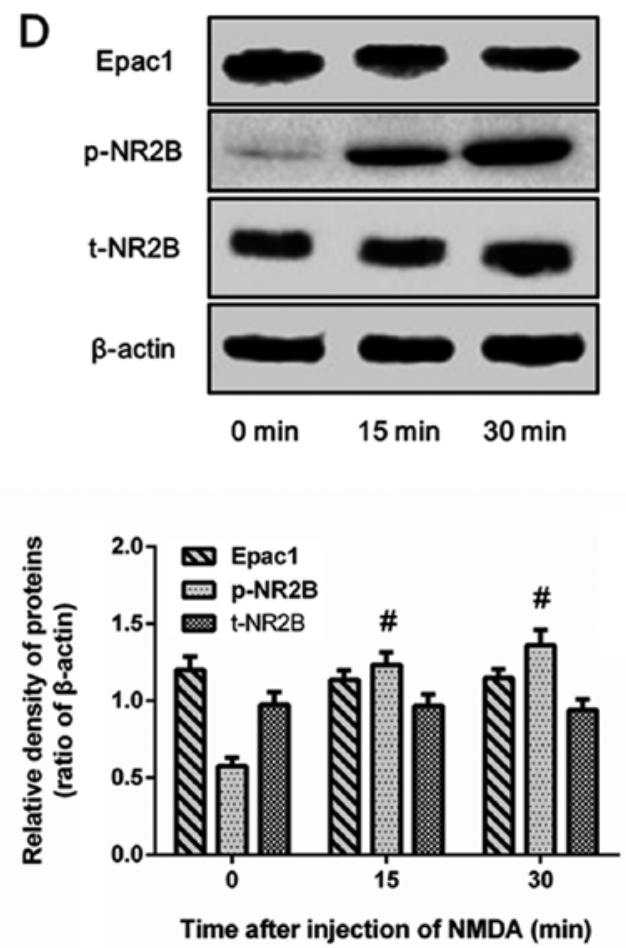

Figure 4. Different effects of antagonist pretreatment on Epac1 or NR2B agonist-induced mechanical allodynia and DRG Piezo2 expression in normal mice. Normal mice were randomly divided in five groups (n=8/group): Pretreated with ifenprodil or saline and 5 min later intraplantar injection of 8-pCPT (ifenprodil/8-pCPT group and saline/8-pCTP group); pretreated with Epac1-ASODN or saline and then intrathecal administration of NMDA (Epac1-ASODN/NMDA group and saline/NMDA group); intrathecal only or intrathecal and intraplantar injection of saline (vehicle group). (A) PWMT was measured at 0,5, 15, 30, 60 and 90 min after injected with agonist. (B-D) Expression levels of Epac1, p-NR2B and Piezo2 in the ipsilateral lumbar DRG L2-L5 segments were detected at the time of 0,15 or 30 min after injection. Data represents means $\pm \mathrm{SD}$. ${ }^{~} \mathrm{P}<0.05$ vs. $0 \mathrm{~min} ;{ }^{*} \mathrm{P}<0.05$ vs. vehicle group at each time point. ${ }^{\# \prime} \mathrm{P}<0.05$ vs. saline group. ASODN, antisense oligodeoxynucleotides; DRG, dorsal root ganglion; PWMT, paw withdrawal mechanical threshold; p-, phosphorylated.

function, Epac1 is unlikely to directly activate Piezo2 (24). Therefore, the underlying mechanism may involve protein kinases or other factors that induce the increase in cytosolic $\mathrm{Ca}^{2+}$.

Previous studies have demonstrated that NMDA receptors (especially NR2B) are essential in the nociceptive processing during BCP $(14,27)$. The activation of NMDA receptors leads to excessive $\mathrm{Ca}^{2+}$ influx or marked increases in cytosolic $\mathrm{Ca}^{2+}$, which triggers a complex cascade of events, including the activation of $\mathrm{Ca}^{2+}$-dependent protein kinases, and further activates a number of downstream effectors $(28,29)$. Previous studies have reported that Epac may exert positive regulatory effects on NMDA receptor activation, which causes an increase in the intracellular $\mathrm{Ca}^{2+}$ levels $(30,31)$. Thus, it was hypothesized that NR2B may be involved in the regulation of Piezo2. In the present study, with the exacerbation or remission of the mechanical allodynia of BCP, NR2B and Piezo2 expression levels exhibited a similar pattern. In addition, pretreatment with the NRB antagonist reversed the increase in Piezo2-mediated mechanical allodynia induced by the Epac1 agonist. However, pretreatment with the Epac1 antagonist followed by the NR2B agonist was not sufficient. These results suggested that NR2B may mediate the Epac1-Piezo2 pathway, contributing to the development of the mechanical allodynia of BCP. 


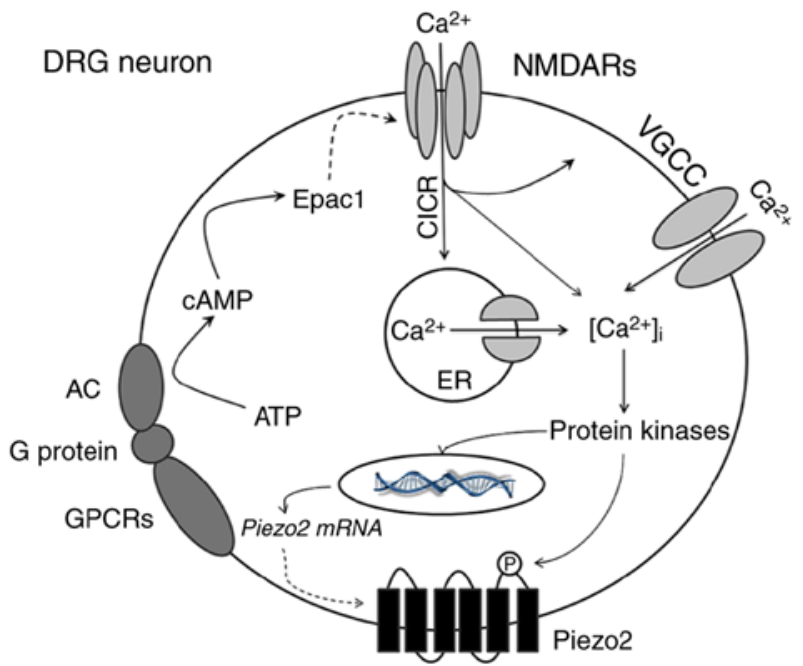

Figure 5. Schematic representation of NR2B-mediated Epac1-Piezo2 signaling pathway possibly participating in the mechanical allodynia of BCP. In the mouse model of BCP, peripheral nociceptive stimulation leads to the activation of GPCRs in DRG neurons which triggers the conversion of ATP into cAMP. As an important second messenger, cAMP directly activates Epac1 protein and then regulates the activity of NR2B-containing NMDA receptors. Activated NMDA receptors cause a significant increase in $\left[\mathrm{Ca}^{2+}\right]_{i}$ through multiple ways, such as the directly influx of calcium ions, CICR and VGCC. Various calcium-dependent protein kinases (such as ERK or CaMK) may be subsequently activated. On the one hand, the synthesis of Piezo2 mRNA or protein is increased; on the other hand, phosphorylation of Piezo2 enhances its function which mediate large amount of cations influx. Based on these mechanisms, the excitability of neurons continues to escalate and ultimately increases the transmission of peripheral nociceptive signals to the center, which leads to the production and maintenance of mechanica hyperalgesia in BCP. GPCRs, G protein-coupled receptors; ATP, adenosine triphosphate; cAMP, cyclic adenosine monophosphate; DRG, dorsal roo ganglion; CICR, calcium-induced calcium release; VGCC, voltage-gated calcium channel; ERK, extracellular-signal regulated kinase; CaMK, calmodulin-dependent kinase.

Certain details of the results of the present study require further illustration. Firstly, the present results suggested that disturbing the Epac1-Piezo2 signaling pathway alleviated the mechanical allodynia of BCP, which was similar to the results of previous studies $(7,32)$. Secondly, behavioral changes are the gold standard for judging the occurrence and development of pain. The proficiency in the establishment of cancer-induced femur pain model and the corresponding imaging and histological changes have been fully illustrated in a previous study (33). Thus, inoculation of osteosarcoma cells into the femur induced progressive mechanical allodynia in this study, indicating the successful establishment of a cancer-induced pain model in mice, similarly to previous studies $(14,34,35)$. Thirdly, the up-down method descripted by Chaplan et al (18) is a classical method of assessing mechanical allodynia. The studies were not only conducted by sequentially increasing and decreasing the stimulus strength, but repeated five times. The lowest von Frey filaments which had three or more positive responses were regarded as PWMT. Hence, the method modified is more practical. In addition, the mechanism of bone cancer pain is complex and many factors are involved at different stages. Therefore, other mechanism involved in mediating Piezo2 and influencing the expression of Epac1 and NR2B on day 7 cannot be excluded. It is a very interesting phenomenon and we will continue to study in depth.
Moreover, the biological activity, the affinity for intended target sequences, and the safety profile of antisense oligodeoxynucleotides (AS-ODNs) cannot be neglected. Future study will be required for validation and translation. Besides, as summarized in Fig. 5, Piezo2 is activated by complicated signal transduction mechanisms and produces mechanically activated cationic current (7). This current further induces strong depolarization in somatosensory neurons and ultimately increases the transmission of peripheral nociceptive signals to the center, which is postulated to lead to the production and maintenance of mechanical hyperalgesia in bone cancer pain. Finally, since BCP possesses the characteristics of inflammatory, neuropathic and tumorigenic components, it is more complicated compared with other forms of pain $(36,37)$; therefore, based on previous studies $(12,16,38,39)$, the present study applied agonists by intraplantar or intrathecal injection to induce mechanical allodynia to partly simulate the mechanical allodynia of BCP. However, further in-depth studies are required to further explore the detailed underlying mechanisms.

In conclusion, the results of the present study demonstrated that the DRG Epac1-Piezo2 signaling pathway may contribute towards mechanical allodynia signal processing in the development of BCP. NR2B, which is a crucial downstream regulator of Epac1, was implicated in the regulation of mechanical allodynia mediated by Epac1-Piezo2 pathway. As an Epac1 inhibitor, Epac1-ASODN alleviated the BCP-induced mechanical allodynia, suggesting a potential application in BCP. Piezo2 may be a valuable clinical target, and specific Piezo2 blockers may be efficient tools for understanding mechanical allodynia and provide an analgesic strategy for clinical therapy.

\section{Acknowledgements}

Not applicable.

\section{Funding}

This study was supported by grants from the National Natural Science Foundation of China (grant nos. 81500951 and 81870875), Six Talent Peaks Project of Jiangsu Province (grant no. YY-077), Young Medical Talents of Jiangsu Province (grant no. QNRC2016014) and Fundamental Research Funds for the Central Universities (grant no. 021414380014).

\section{Availability of data and materials}

The analyzed datasets generated during the study are available from the corresponding author on reasonable request.

\section{Authors' contributions}

$\mathrm{KN}$, WZ and ZM conceived and designed the experiments. YN and YM participated in the behavior test, western blotting and RT-qPCR. YW carried out the collection of tissues. XG performed the design of study and coordination. $\mathrm{KN}$ and $\mathrm{WZ}$ confirm the authenticity of all the raw data. All authors read and approved the final manuscript. 


\section{Ethics approval and consent to participate}

All animal experimental procedures were carried out in accordance with the guidelines of the National Institutes of Health Guide for Care and were approved by the Medical College of Nanjing University Animal Care and Use Committee (approval no. 20210122; Nanjing, China).

\section{Patient consent for publication}

Not applicable.

\section{Competing interests}

The authors declare that they have no competing interests.

\section{References}

1. Mantyh P: Bone cancer pain: Causes, consequences, and therapeutic opportunities. Pain 154 (Suppl 1): S54-S62, 2013.

2. Scarborough BM and Smith CB: Optimal pain management for patients with cancer in the modern era. CA Cancer J Clin 68 : 182-196, 2018.

3. Colvin L and Fallon M: Challenges in cancer pain management-bone pain. Eur J Cancer 44: 1083-1090, 2008.

4. Coste B, Mathur J, Schmidt M, Earley TJ, Ranade S, Petrus MJ, Dubin AE and Patapoutian A: Piezo1 and Piezo2 are essential components of distinct mechanically activated cation channels. Science 330: 55-60, 2010

5. Ranade SS, Woo SH, Dubin AE, Moshourab RA, Wetzel C, Petrus M, Mathur J, Bégay V, Coste B, Mainquist J, et al: Piezo2 is the major transducer of mechanical forces for touch sensation in mice. Nature 516: 121-125, 2014.

6. Murthy SE, Loud MC, Daou I, Marshall KL, Schwaller F, Kühnemund J, Francisco AG, Keenan WT, Dubin AE, Lewin GR and Patapoutian A: The mechanosensitive ion channel Piezo2 mediates sensitivity to mechanical pain in mice. Sci Transl Med 10: eaat9897, 2018.

7. Szczot M, Liljencrantz J, Ghitani N, Barik A, Lam R Thompson JH, Bharucha-Goebel D, Saade D, Necaise A, Donkervoort S, et al: PIEZO2 mediates injury-induced tactile pain in mice and humans. Sci Transl Med 10: eaat9892, 2018.

8. Berkey SC, Herrera JJ, Odem MA, Rahman S, Cheruvu SS, Cheng X, Walters ET, Dessauer CW and Bavencoffe AG: EPAC1 and EPAC2 promote nociceptor hyperactivity associated with chronic pain after spinal cord injury. Neurobiol Pain 7: 100040, 2019.

9. Huang LY and Gu Y: Epac and nociceptor sensitization. Mol Pain 13: $1744806917716234,2017$.

10. Li ZH, Cui D, Qiu CJ and Song XJ: Cyclic nucleotide signaling in sensory neuron hyperexcitability and chronic pain after nerve injury. Neurobiol Pain 6: 100028, 2019.

11. Hucho TB, Dina OA and Levine JD: Epac mediates a cAMP-to-PKC signaling in inflammatory pain: An isolectin B4(+) neuron-specific mechanism. J Neurosci 25: 6119-6126, 2005.

12. Eijkelkamp N, Linley JE, Torres JM, Bee L, Dickenson AH, Gringhuis M, Minett MS, Hong GS, Lee E, Oh U, et al: A role for Piezo2 in EPAC1-dependent mechanical allodynia. Nat Commun 4: 1682, 2013.

13. Zimmermann M: Ethical guidelines for investigations of experimental pain in conscious animals. Pain 16: 109-110, 1983.

14. Ni K, Zhou Y, Sun YE, Liu Y, Gu XP and Ma ZL: Intrathecal injection of selected peptide Myr-RC-13 attenuates bone cancer pain by inhibiting KIF17 and NR2B expression. Pharmacol Biochem Behav 122: 228-233, 2014.

15. Schwei MJ, Honore P, Rogers SD, Salak-Johnson JL, Finke MP, Ramnaraine ML, Clohisy DR and Mantyh PW: Neurochemical and cellular reorganization of the spinal cord in a murine model of bone cancer pain. J Neurosci 19: 10886-10897, 1999.

16. Chen G, Xie RG, Gao YJ, Xu ZZ, Zhao LX, Bang S, Berta T, Park CK, Lay M, Chen W and Ji RR: $\beta$-arrestin-2 regulates NMDA receptor function in spinal lamina II neurons and duration of persistent pain. Nat Commun 7: 12531, 2016.
17. Xiaoping G, Xiaofang Z, Yaguo Z, Juan Z, Junhua W and Zhengliang M: Involvement of the spinal NMDA receptor/PKC $\gamma$ signaling pathway in the development of bone cancer pain. Brain Res 1335: 83-90, 2010

18. Chaplan SR, Bach FW, Pogrel JW, Chung JM and Yaksh TL: Quantitative assessment of tactile allodynia in the rat paw. J Neurosci Methods 53: 55-63, 1994.

19. Livak KJ and Schmittgen TD: Analysis of relative gene expression data using real-time quantitative PCR and the 2(-Delta Delta C(T)) method. Methods 25: 402-408, 2001.

20. Luger NM, Mach DB, Sevcik MA and Mantyh PW: Bone cancer pain: From model to mechanism to therapy. J Pain Symptom Manage 29 (5 Suppl): S32-S46, 2005.

21. Goblirsch MJ, Zwolak PP and Clohisy DR: Biology of bone cancer pain. Clin Cancer Res 12: 6231s-6235s, 2006.

22. Krames ES: The role of the dorsal root ganglion in the development of neuropathic pain. Pain Med 15: 1669-1685, 2014.

23. Hucho T and Levine JD: Signaling pathways in sensitization: Toward a nociceptor cell biology. Neuron 55: 365-376, 2007.

24. Bos JL: Epac proteins: Multi-purpose cAMP targets. Trends Biochem Sci 31: 680-686, 2006.

25. Bagriantsev SN, Gracheva EO and Gallagher PG: Piezo proteins: Regulators of mechanosensation and other cellular processes. J Biol Chem 289: 31673-31681, 2014.

26. Wang L, Zhou H, Zhang M, Liu W, Deng T, Zhao Q, Li Y, Lei J, Li X and Xiao B: Structure and mechanogating of the mammalian tactile channel PIEZO2. Nature 573: 225-229, 2019.

27. Gu X, Zhang J, Ma Z, Wang J, Zhou X, Jin Y, Xia X, Gao Q and Mei F: The role of N-methyl-D-aspartate receptor subunit NR2B in spinal cord in cancer pain. Eur J Pain 14: 496-502, 2010.

28. Lau CG, Takeuchi K, Rodenas-Ruano A, Takayasu Y, Murphy J, Bennett MVL and Zukin RS: Regulation of NMDA receptor Ca2+ signalling and synaptic plasticity. Biochem Soc Trans 37: 1369-1374, 2009

29. Liu XG and Zhou LJ: Long-term potentiation at spinal C-fiber synapses: A target for pathological pain. Curr Pharm Des 21: 895-905, 2015.

30. Sánchez-Pérez AM, Llansola M and Felipo V: Modulation of NMDA receptors by AKT kinase. Neurochem Int 49: 351-358, 2006.

31. Gonzalez-Robayna IJ, Falender AE, Ochsner S, Firestone GL and Richards JS: Follicle-Stimulating hormone (FSH) stimulates phosphorylation and activation of protein kinase $\mathrm{B}$ (PKB/Akt) and serum and glucocorticoid-lnduced kinase (Sgk): Evidence for A kinase-independent signaling by FSH in granulosa cells. Mol Endocrinol 14: 1283-1300, 2000.

32. Singhmar P, Huo X, Eijkelkamp N, Berciano SR, Baameur F, Mei FC, Zhu Y, Cheng X, Hawke D, Mayor F Jr, et al: Critical role for Epacl in inflammatory pain controlled by GRK2-mediated phosphorylation of Epac1. Proc Natl Acad Sci USA 113: 3036-3041, 2016.

33. Ren BX, Gu XP, Zheng YG, Liu CL, Wang D, Sun YE and Ma ZL: Intrathecal injection of metabotropic glutamate receptor subtype 3 and 5 agonist/antagonist attenuates bone cancer pain by inhibition of spinal astrocyte activation in a mouse model. Anesthesiology 116: 122-132, 2012.

34. Yang Y, Zhang J, Gao Q, Bo J and Ma Z: Etanercept attenuates thermal and mechanical hyperalgesia induced by bone cancer. Exp Ther Med 13: 2565-2569, 2017.

35. Sun Y, Jiang M, Hou B, Lu C, Lei Y, Ma Z and Gu X: Mas-related gene (Mrg) C activation attenuates bone cancer pain via modulating Gi and NR2B. PLoS One 11: e0154851, 2016.

36. Mercadante $S$ and Fulfaro F: Management of painful bone metastases. Curr Opin Oncol 19: 308-314, 2007.

37. Basbaum AI, Bautista DM, Scherrer G and Julius D: Cellular and molecular mechanisms of pain. Cell 139: 267-284, 2009.

38. Li S, Cao J, Yang X, Suo ZW, Shi L, Liu YN, Yang HB and Hu XD: NR2B phosphorylation at tyrosine 1472 in spinal dorsal horn contributed to N-methyl-D-aspartate-induced pain hypersensitivity in mice. J Neurosci Res 89: 1869-1876, 2011.

39. Alvarez-Vega M, Baamonde A, Gutiérrez M, Hidalgo A and Menéndez L: Intrathecal N-methyl-D-aspartate (NMDA) induces paradoxical analgesia in the tail-flick test in rats. Pharmacol Biochem Behav 65: 621-625, 2000. 